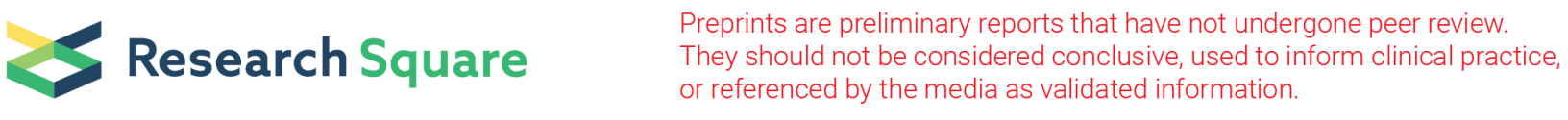

\title{
Predictors for Outcomes related to upper Extremity Musculoskeletal Disorders in a healthy Working Population: a methodological Approach
}

\author{
Oliver Lotter ( $\nabla$ oliver.lotter@googlemail.com ) \\ Klinikum Landkreis Tuttlingen https://orcid.org/0000-0002-7053-2294 \\ Tobias Lieb \\ Office for Occupational and Hand Therapy \\ Jochen Molsner \\ IAS-Group for Occupational Health Management \\ Viktor Breul \\ Department of Medical Scientific Affairs
}

\section{Research article}

Keywords: Work-related musculoskeletal disorders (WMSD), upper extremity, repetitive work, surgical device mechanics, DASH score, Purdue Pegboard Test, multivariate analysis, correlation, predictors

Posted Date: September 22nd, 2020

DOI: https://doi.org/10.21203/rs.3.rs-73555/v1

License: (9) (1) This work is licensed under a Creative Commons Attribution 4.0 International License. Read Full License 


\section{Abstract}

\section{Background}

To investigate the association between different clinical endpoints and the presence of upper extremity work-related musculoskeletal disorders (WMSDs) in a healthy working population. Furthermore, the influence of socio-demographic, workrelated and individual predictors on different endpoints was examined.

Methods

Two self-completion questionnaires were administered to 70 workers and employees. In addition, a standardized physical examination and an industry test were performed in this cross-sectional study. Correlations between WMSDs and clinical endpoints were analysed with the Spearman method. Depending on the type of dependent endpoint, linear or logistic multivariate regression models were used to study the strength of associations with a pre-defined set of potential influencing factors.

Results

The prevalence of WMSDs was 56\% (39/70). Correlations between WMSDs and the DASH score / pain under strain (VAS) were by far the strongest ones. Independent predictors could not be identified as risk factors for WMSDs, but there was some correlation between these factors.

\section{Conclusions}

The DASH score, used in the primary analysis of the study data, remains a close candidate for best surrogate endpoint for WMSD detection. The VAS has to be examined for this role in further research. Our analysis should help to improve the methodological quality of future occupational health studies through improved standards.

Trial registration

This study was registered at ClinicalTrials.org with the identifier NCT03014128, on January 9, 2017.

\section{Background}

Work-related musculoskeletal disorders (WMSD), also known as cumulative trauma disorders (CTD), repetitive strain injuries (RSI) or occupational overuse syndromes (OOS), are pathologies of multifactorial origin in which the work environment contributes significantly to the condition and/or the condition is aggravated by the work activity [1]. Such diseases are caused by the accumulation of microtraumatic events in the musculoskeletal system over a long period of time and represent a broad spectrum of inflammatory and degenerative diseases jeopardising the quality of life and functional capacity of those affected $[2,3]$. They are a significant occupational health problem among industrial and clerical workers with a strong medical, economic, and social impact in terms of absences due to sickness and early retirement, cost of medical care, lost production, and personal suffering [4]. These disorders represent one of the greatest work-related challenges of our time with great impact on individuals and society.

WMSDs account for more than $48 \%$ of work-related disorders and diseases $[5,6]$. The overall global prevalence for such conditions ranges from $4-30 \%$ increasing with age, and the annual prevalence lies between $0.14 \%$ and $14.9 \%$ across different industries and work processes [7-10]. Treatment costs for WMSDs are estimated at $1.3 \%$ of the US gross national product and between $0.5 \%$ and $2.0 \%$ in Scandinavian countries $[11,12]$.

The multifactorial genesis for WMSDs has been shown to be linked to physical / biomechanical, psychosocial, organisational and genetic factors, which are associated with numerous professional situations and specific industries [13-15]. Studies can 
only consider some of these risk factors simultaneously, which makes it difficult to make a statement about the influence of competing factors [16].

Furthermore, the study designs and research methods used to collect and process data via WMSDs are extremely heterogeneous. Our literature search revealed that out of 262 original research articles, only about $4 \%$ of the studies contained a clinical examination of the subjects with or without structured questionnaires / interviews including a control group [17].

The aim of this analysis was to investigate the association between different clinical endpoints which are likely to be measured in the clinical routine, but usually just represent surrogates for an actual health problem, and the presence of upper extremity WMSDs in a healthy working population. It also intended to take into account potentially predisposing sociodemographic, work-related and individual characteristics ("risk factors").

\section{Methods}

\section{Study Population}

The study was conducted at the headquarters and main production site of Aesculap AG, Tuttlingen / Germany with about 3500 workers and employees. This company is the world market leader in the field of surgical instruments. The study population was divided into three groups based on their occupational activities: group I = grinding and polishing characterised by repetitive and forceful exertions ("grinding"), group II = inspection and packaging characterised by repetitive exertions without force ("packaging"), and group III = all other white-collar and blue-collar employees as a cross-section of the company as a control group ("control").

\section{Study design and data collection}

Random samples of active white-collar and blue-collar workers were drawn from the three groups using statistic software and recruited between September 2017 and March 2018. No incentives were offered. Eligibility criteria were applied according to our previous publication [17]. The participants were asked to fill in two standardised self-administered questionnaires. The first questionnaire obtained demographic and personal data, considered as predictors in the analyses, such as sex, handedness, secondary occupation, sporting and physical hobbies, age, height and weight (body mass index), volume of employment, and years of service.

\section{Clinical endpoints and signs for WMSDs}

\section{$\underline{\text { DASH score }}$}

As second questionnaire, the validated Disabilities of the Arm, Shoulder and Hand (DASH) outcome measure was applied to assess physical function and symptoms $[18,19]$. The results of this 30 -item questionnaire were used to calculate a scale score ranging from 0 (no disability) to 100 (most severe disability).

\section{$\underline{\text { VAS }}$}

Instrument to measure the intensity of pain on a Visual Analogue Scale. A Likert scale between 1 (no pain) and 10 (maximal pain) points was used to collect the subjective evaluation for pain at rest and pain under strain. Results were analysed as continuous parameters.

$\underline{\mathrm{ROM}}$

Range of motion (ROM) measurements of wrist joint mobility of both hands were performed using a goniometer in the three planes extension / flexion (E/F), supination / pronation (S/P) and ulnar / radial abduction (U/R) according to the neutral zero method [20]. 
Grip strength

Measurement of the maximum hand force using the Jamar dynamometer in three consecutive passes per side.

$\underline{\text { PPB Test }}$

The neurophysiological Purdue Pegboard (PPB) Test was performed to determine the dexterity of the participants [21]. As a former industrial test, it now serves primarily to assess disabilities and limitations. The pegboard consists of a board with two parallel rows of 25 holes each into which cylindrical metal pegs are placed by the examinee. The test involves a total of four trials [22]. The subsets for preferred, non-preferred, and both hands require the test person to place the pins in the holes as quickly as possible, with the score being the number of pins placed in $30 \mathrm{sec}$. Purdue Pegboard trial number 4 was chosen as the representative clinical endpoint as it summarises trials numbers 1 to 3 well by adding them up, and therefore should show the potential differences more clearly.

\section{Subjective complaints}

The presence of subjective complaints on the upper extremity of a test person was assessed in the form of a yes / no type of self-reported question.

\section{Clinical signs of WMSDs}

Musculoskeletal diagnoses were measured via a structured physical examination of the elbow, forearm, wrist, and hand by one single hand surgeon. The examiner was blinded to the questionnaire responses of the test persons. The diagnoses of

- De Quervain's tenosynovitis

- Tennis elbow

- Nerve entrapment syndromes including

- carpal tunnel syndrome (median nerve)

- cubital tunnel syndrome and Guyon's canal syndrome (ulnar nerve)

were made based on pathognomonic clinical signs for upper extremity pathologies, after selection by a multidisciplinary team consisting of an occupational physician, hand surgeon and occupational therapist [23]. These included pressure pain on the radial side of the wrist together with Finkelstein's test for De Quervain's tenosynovitis, lateral epicondyle pain and Maudsley's test for tennis elbow, and the combination of Hoffman-Tinel sign and static two-point discrimination (2-PD) for finger sensibility for nerve entrapment syndromes, adding Phalen's test specific for Carpal tunnel syndrome [24-27]. The presence of any of the above diagnoses in a study participant was considered to be a WMSD diagnosis, which served as gold standard for correlation analyses with other clinical endpoints and predictors.

\section{Statistical Analysis}

The study was initially powered for the proof of the hypothesis of clinical equivalence of occupational groups in regard to the DASH score [17]. This hypothesis could be confirmed by primary analysis.

\section{Correlations}

In the current analysis, correlations between variables were assessed using Spearman correlation coefficients and their $\mathrm{p}$ values. Prior to the calculation of correlation coefficients, bivariate scatter plots were visually examined in order to investigate interrelationships between the clinical endpoints. Prediction ellipses were applied to the scatter plotsBecause the ellipse is centred at the two-dimensional mean and expanded to cover the maximal part of the data points, it can visually indicate the strength of interrelation as well as outliers in the data. A stretched tilted ellipse indicates highly correlated variables, whereas an ellipse that is nearly circular indicates little correlation. 
A set of potential predictors influencing clinical endpoints (i.e. independent variables or effects) was chosen based on the clinical considerations. Six variables were selected for this role:

- gender (female / male)

- body mass index (BMI)

- occupation group (grinding / packaging / control)

- secondary occupation and/or physical hobbies

- age

- years in service.

A multivariate regression modelling of clinical endpoints was applied in order to identify their significant predictors, based on the p-value of the effect. Linear or logistic regression was used dependent on a respective continuous or dichotomous type of the endpoint variable.

\section{$\underline{\text { Repeated measurements }}$}

Some endpoints in our selection enclose repeated measurements in the same subject like left- and right-hand measurements of hand force or range of motion. Additionally, the repetition scheme may have included 3 subsequent attempts with each arm (hand force) or a recording of 3 dimensions (E/F, S/P, U/R) for range of motion. Adequate use of such repeated data structures required consideration of more sophisticated repeat measurement modelling methods, rather than just using the mean of the three attempts, in order to avoid loss of information and statistical power.

\section{Handedness}

Considering handedness was more challenging than it appears. The study population included four types as follows:

\begin{tabular}{|lll|}
\hline & N & $\%$ \\
\hline Total & 70 & 100 \\
\hline Dominant hand & & \\
\hline left & 6 & 8.57 \\
\hline left mixed & 6 & 8.57 \\
\hline right & 55 & 78.57 \\
\hline right mixed & 3 & 4.29 \\
\hline
\end{tabular}

For analytic purposes, left and right-handed subjects were put together with their corresponding mixed types.

Measurements referring to the right or left body side were analyzed as referring to the primary or to the non-primary hand in order to evaluate the effect of the handedness.

\section{Descriptive statistics}

Mean values and standard deviations (SD) in brackets were described with approximately normally distributed continuous data. Median and interquartile ranges in brackets were shown for non-normally distributed continuous data [28]. We calculated absolute and relative frequencies for categorical variables. Numerators and denominators of the calculations were always given in parenthesis when reporting percentages in categorical data. 
As the study sample of 70 subjects might lack power to detect small effects, the regression results must be considered with caution. The classic 5\% significance level does not seem appropriate for exploration purposes, so effects showing p-values up to 0.1 will be closely considered. This procedure follows our aim to figure out contributors to clinical endpoints for future studies rather than provide evidence for correlations in our study sample.

SAS software version 9.4 with Enterprise Guide $7.1 \mathrm{GUI}$ was used for analyses.

\section{Results}

\section{Recruitment and baseline characteristics}

The total population consisted of 63 persons in group I, 208 in group II and 2501 in group III. Random samples were drawn from these groups after completion of the questionnaires and proving eligibility, so that a total of 70 subjects (grinding $n=20$, packaging $n=24$, control $n=26)$ were included in the study. The subjects were predominantly men $(67 \%(47 / 70))$ and righthanded individuals (83\% (55/70)). Only few had a secondary occupation (9\% (6/70)) and 61\% (43/70) reported having sporting or physical hobbies. The three groups had comparable demographic data with regard to age (42.1 (12.2) years), body mass index (BMI) $\left(26.2(5.0) \mathrm{kg} / \mathrm{m}^{2}\right)$, full volume of employment $(91 \%(64 / 70))$ and years of service at the company (16.1 (9 to 28) years). For the study population flowchart and detailed demographic data in the individual groups, please refer to our previous publication [17].

\section{Clinical endpoints}

The DASH score, clinical parameters and the Purdue Pegboard (PPB) Test score are shown in Table 1. Our mean scores are in good agreement with the normative DASH score [29]. Note that lower DASH scores are associated with a better situation. Three DASH questionnaires were excluded from the analysis because of incompleteness. We did not expect any relevant differences between the grip strength of the right and left hands, therefore we used the parameter dominant / non-dominant hand for further analysis. When comparing grip strength with reference values from a healthy population, subdivided according to sex and age group, below-average values were found [30]. This was also the case with the PPB test [31].

Table 1

Measured continuous clinical endpoints

\begin{tabular}{|c|c|c|c|}
\hline & $\mathbf{N}$ & measured values & reference values \\
\hline & & mean (SD) & mean (SD) \\
\hline DASH score & 67 & $9.45(8.96)$ & $10.1(14.7)$ \\
\hline VAS at rest (points) & 70 & $1.61(1.45)$ & - \\
\hline VAS under strain (points) & 70 & $2.53(2.20)$ & - \\
\hline ROM E/F (degrees) & 70 & $124.89(13.68)$ & - \\
\hline ROM S/P (degrees) & 70 & $177.68(7.08)$ & - \\
\hline ROM U/R (degrees) & 70 & $50.96(3.36)$ & - \\
\hline Grip strength $(\mathrm{kg})-$ male & 70 & $47.75(13.55)$ & $54(7)$ \\
\hline Grip strength $(\mathrm{kg})$-female & 70 & $27.33(4.93)$ & $32(6)$ \\
\hline PPB Test (points) & 70 & $41.03(4.83)$ & $43.21(5.21)^{\star}$ \\
\hline \multicolumn{4}{|c|}{$\begin{array}{l}\text { DASH = Disabilities of the Arm, Shoulder and Hand, VAS = pain on Visual Analogue Scale, } \mathrm{ROM}=\text { range of motion, } \mathrm{E} / \mathrm{F}= \\
\text { extension } / \text { flexion, } \mathrm{S} / \mathrm{P}=\text { supination } / \text { pronation, } \mathrm{U} / \mathrm{R}=\mathrm{ulnar} / \mathrm{radial} \text { abduction, } \mathrm{PPB} \text { Test }=\text { Purdue Pegboard Test. } \\
{ }^{*} \text { Calculated for the mean age category }(40-49 \text { years }) \text { and for the study population gender ratio }(23 / 70 \text { female) according } \\
\text { to [31]. }\end{array}$} \\
\hline
\end{tabular}


Subjective complaints (i.e. symptoms) were present in 47\% (33/70) and pathognomonic clinical signs for upper extremity WMSDs (i.e. diagnoses) at the elbow, forearm and/or wrist (trigger finger, Finkelstein's test, Maudsley's test, Hoffman-Tinel sign, and Phalen's test) were found in $56 \%$ (39/70) of our collective (Table 2). Bilateral manifestation was present in $34 \%$ (24/70), and $14 \%(10 / 70)$ of the subjects had two or more different pathologies in the ipsilateral limb. In the case of a positive Hoffmann-Tinel sign, $91 \%(21 / 23)$ of these were located at the medial elbow as a sign of ulnar tunnel syndrome.

Table 2

Measured categorical clinical signs

\begin{tabular}{|l|c|}
\hline & $\begin{array}{c}\text { total subjects } \\
\mathbf{N}=70)\end{array}$ \\
\hline Subjective complaints * & $47 \%(33 / 70)$ \\
\hline Trigger finger & $10 \%(7 / 70)$ \\
\hline Finkelstein's test & $10 \%(7 / 70)$ \\
\hline Maudsley's test (middle finger test) & $14 \%(10 / 70)$ \\
\hline Hoffman-Tinel sign & $33 \%(23 / 70)$ \\
\hline Phalen's test & $10 \%(7 / 70)$ \\
\hline * Counts among the clinical endpoints without being a clinical sign, listed in this table for categorical properties \\
\hline
\end{tabular}

\section{Correlation of WMSDs with other clinical endpoints}

The relationship between WMSDs and the DASH score is shown as an example using a scatter plot with a prediction ellipse (Fig. 1) [32]. Further correlations between grip strength and ROM E/F, and between VAS at rest and under strain, DASH and subjective complaints became apparent.

A closer look into the scatter plot reveals an obvious bottom effect in the DASH distribution. DASH values of probands with WMSD concentrate more in the 10-point range, whereas the "no WMSD"-group very often has a DASH score of 0 . This causes the slight tilt of the ellipse, correlating with the Pearson correlation coefficient, which, in the first approximation visualizes an interrelation between the DASH score and WMSDs. However, the non-normality contradicts the application of the parametric Pearson regression and causes a switch to the non-parametric Spearman correlation. Its coefficient is indeed much more sensitive to this effect, due to its robustness towards non-normal distribution of the DASH values. For the same reason, the normality assumption is not given for ROM and VAS distributions, so the Spearman method has to be preferred for evaluation of interactions between WMSDs and these endpoints.

Bivariate correlation coefficients between clinical endpoints and the diagnosis of WMSDs are shown in Table 3. Only two of the collected clinical endpoints were associated with a p-value below 0.1 (presented in bold letters): the DASH score and VAS under strain. Both endpoints are positively correlated with WMSDs, meaning that higher scores are associated with a WMSD diagnosis. These results also make sense from a clinical point of view since both DASH scores and VAS associate higher scores with worse outcomes. 
Table 3

Correlation between WMSDs and other clinical endpoints

\begin{tabular}{|lll|}
\hline Clinical endpoint & $\mathbf{N}$ & Spearman $\mathbf{r}$ \\
\hline DASH score & 67 & $0.23(\mathbf{p}=\mathbf{0 . 0 5 6})$ \\
\hline VAS at rest & 70 & $0.04(p=0.755)$ \\
\hline VAS under strain & 70 & $0.26(\mathbf{p}=0.029)$ \\
\hline ROM E/F & 70 & $-0.01(p=0.943)$ \\
\hline ROM S/P & 70 & $-0.17(p=0.168)$ \\
\hline ROM U/R & 70 & $-0.12(p=0.316)$ \\
\hline Grip strength & 70 & $0.13(p=0.269)$ \\
\hline PPB Test & 70 & $0.03(p=0.787)$ \\
\hline Subjective complaints & 70 & $0.15(p=0.213)$ \\
\hline $\begin{array}{l}\text { DASH = Disabilities of the Arm, Shoulder and Hand, VAS = pain on Visual Analogue Scale, ROM = range of motion, E/F = } \\
\text { extension/flexion, S/P = supination/pronation, U/R = ulnar/radial abduction, PPB Test = Purdue Pegboard Test }\end{array}$ \\
\hline
\end{tabular}

\section{Multivariate regression analyses of predictors}

The relationship between the pre-specified predictors and the occurrence of WMSDs was investigated through the analysis of a binary logistic regression (Table 4). None of these factors seem to be a risk factor for WMSDs, the minimal p-value was 0.14 .

Table 4

Results of the multivariate analysis for the endpoint WMSDs

\begin{tabular}{|ll|}
\hline Effect/factor & p-value \\
\hline Gender (female/male) & 0.14 \\
\hline Body mass index (BMI) & 0.41 \\
\hline Occupation group (grinding/packaging/control) & 0.43 \\
\hline Secondary occupation and/or physical hobbies & 0.57 \\
Age & 0.96 \\
Years in service & 0.99 \\
\hline
\end{tabular}

The respective analyses following the same layout were performed using other clinical endpoints, with a linear instead of logistic regression applied in case of continuous endpoints. Results are shown in Table 5. Only the combinations with a pvalue of $p \leq 0.1$ are listed. $p$-values below the level of 0.05 are presented in bold letters. 
Table 5

Results of the multivariate analysis of secondary clinical endpoints

\begin{tabular}{|c|c|c|}
\hline Basic endpoint & Effect/factor & p-value \\
\hline DASH & gender (female/male) & 0.0190 \\
\hline \multirow[t]{3}{*}{ ROM } & dimension of movement (E/F, S/P or U/R) & $<0.0001$ \\
\hline & years in service & 0.0213 \\
\hline & gender (female/male) & 0.0350 \\
\hline \multirow[t]{2}{*}{ Subjective complaints } & age & 0.0424 \\
\hline & years in service & 0.0599 \\
\hline VAS at rest & BMI & 0.0914 \\
\hline VAS under strain & $\mathrm{BMI}$ & 0.0272 \\
\hline \multirow[t]{5}{*}{ Grip strength } & gender (female/male) & $<0.0001$ \\
\hline & age & 0.0002 \\
\hline & $\mathrm{BMI}$ & 0.0003 \\
\hline & secondary occupation and/or physical hobbies & 0.0408 \\
\hline & Years in service & 0.0525 \\
\hline \multicolumn{3}{|c|}{$\begin{array}{l}\text { DASH = Disabilities of the Arm, Shoulder and Hand, } \mathrm{ROM}=\text { range of motion, VAS = pain on Visual Analogue Scale, } \mathrm{BMI}= \\
\text { Body Mass Index, E/F = extension / flexion, } \mathrm{S} / \mathrm{P}=\text { supination } / \text { pronation, } \mathrm{U} / \mathrm{R}=\text { ulnar } / \text { radial abduction }\end{array}$} \\
\hline \multicolumn{3}{|c|}{$\begin{array}{l}\text { In addition to known and obvious correlations such as gender dependence for DASH with higher values in women, or } \\
\text { higher grip strength in men, there was a negative correlation between ROM and years in service, more frequent subjective } \\
\text { complaints of the upper extremities with increasing age, pain under strain on the VAS at a higher BMI, and higher grip } \\
\text { strength when secondary occupation and/or physically demanding hobbies were performed [33]. }\end{array}$} \\
\hline
\end{tabular}

A statistical effect may be observed in Table 5 with by far the lowest p-values concentrated in the analysis of grip strength and, to some extent, of the ROM. This is not really surprising as there were 3 repetitions recorded for every measurement. The additional information gave the analysis of variance more statistical power i.e. more sensitivity for effects detection.

\section{Discussion}

We chose to survey a population of actively employed surgical device mechanics and compared them with a group of employees believed not to be exposed to repetitive hand and arm movements to such a large extent.

The overall prevalence of subjective upper extremity complaints (i.e. symptoms) was $47 \%$ (33/70). Eight of 20 (40\%) grinders, $14 / 24$ (58\%) packers, and 11/26 (42\%) people in the control group reported such symptoms. One or more upper extremity WMSDs (i.e. diagnoses) at the elbow, forearm and/or wrist were found in 56\% (39/70), of which 12/20 (70\%) grinders, 14/24 (54\%) packers and 13/26 (42\%) control persons. These results are consistent with previous studies showing a prevalence rate of symptoms between $21 \%$ and $71 \%$ in the study group and between $6 \%$ and $50 \%$ in the control group [34-46]. With regard to diagnoses, existing studies report a prevalence between $21 \%$ and $56 \%$ in the study group compared to $5-22 \%$ in the control group [35, 36, 38, 39, 40,42]. Within the framework of the standardized clinical examination, we applied rather "softer" criteria, as recommended by Vikari-Juntura [47]. This might explain the higher detection of WMSDs via examination as compared to the lower number of subjective complaints reported in the questionnaire. An above average co-occurrence of medial epicondylitis (golfer's elbow) and nerve entrapment at the medial elbow (cubital tunnel syndrome) was found in $30 \%$ of our test persons and $54 \%$ of the subjects with upper extremity complaints in this cohort [48-50]. 
The clinical endpoint values of our study population were largely consistent with the reference values of the general population, but in some cases (grip strength, PPB tests) also showed below average values. This is surprising and contradicts the study situation, as our cohort tends to have an above-average physical load [51]. Reasons could be mechanical support, shorter working hours and a historical shift in populations' reference values without the first two points mentioned.

The aim of our analysis was to evaluate different clinically established endpoints representing surrogates for an actual health problem, for association with upper extremity WMSDs in terms of a technical evaluation of the survey methods. We also investigated the influence of socio-demographic, work-related and individual independent predictor variables as potential risk factors for WMSDs. The methodological approach is intended to be a proposal for a standardized procedure for future crosssectional studies of this kind.

In the bivariate analysis we found a correlation between the DASH score and WMSDs as well as VAS under strain and WMSDs. A simple clinical explanation for this could be that pathology (WMSDs) manifests itself through pain, especially when the hand is used forcefully. This aspect is a common feature of the DASH questionnaire and also manifests itself with VAS under strain. VAS at rest, ROM, grip strength, PPB Test and the indication of subjective complaints by the study participants were not suitable to detect WMSDs in our study. However, the question of subjective complaints is a central component of many studies and the basis for their interpretation.

Regarding the upper extremity, the validity of questionnaires for WMSDs has not been clarified and it is not known how an optimal questionnaire can be constructed and what information can be obtained [52]. A purely technical investigation using measurement data without clinical examination would have the advantage of resource optimization but could not be related to WMSDs either [53]. This is also supported by the relatively weak correlation between measured clinical endpoints and WMSDs in our study. Accordingly, a clinical examination based on a predetermined set of diagnostic criteria remains the gold standard for cross-sectional investigations in order to keep well-defined disorders separate from more diffuse conditions. Although the clinical examination is time consuming and hard for both the subject and the examiner, it seems to be necessary so far to detect defined WMSDs.

Although the $p$-value has not quite reached the conventional significance level of 0.05 in our study $(p=0.056)$, the correlation we found between WMSDs and the DASH score may not therefore be considered non-existent and could be of interest for the design of future studies [54]. The validated DASH score as a self-administered, region-specific outcome instrument for upperextremity disability and symptoms, was tested against the gold standard of WMSD detection (i.e. the clinical examination). To our knowledge, there are no studies focusing on the correlation between WMSDs and the DASH score, whereas this is the case for some upper extremity pathologies other than WMSDs [55]. According to our analysis, the DASH score has the potential to replace the resource-intensive clinical examination as a screening tool. In case of conspicuous DASH scores, the latter could be used in a focused manner for diagnosis, with therapeutic and preventive measures derived from it. In comparison, the Nordic Musculoskeletal Questionnaire (NMQ), often used in cross-sectional studies, is a simple validated questionnaire that refers to complaints in 9 body parts, including the hand/wrist/elbow [56]. Its content is in no way comparable to the detailed questions of the DASH with its focus on the upper extremity, and hardly exceeds the yes/no question on subjective complaints in our study. To what extent further questionnaires are suitable for the detection of upper extremity pathologies will be the subject of future studies.

For the endpoint WMSD, the multivariate analysis of our study did not show any independent predictors significant at a 0.1level. This is partially in contrast to previous studies, in which work-related and sociodemographic characteristics have been determined as predisposing upper extremity disorders [34]. The first include static postures, excessive force and strain, vibration, repeated pushing, pulling and lifting, overuse of particular anatomical structures or regions, poor posture or improper positioning, awkward movements, long duration of pressure, rapid work pace, short recovery periods, low decision latitude, years of service and job satisfaction [57-61]. Socio-demographic characteristics predicting WMSDs include factors like sex, age, marital status, work experience, body mass index and physical activities [62-67]. It is advisable to select the characteristics of personal factors, physical body functions, environmental factors and mental body functions based on the 
International Classification of Functioning, Disability and Health (ICF) [68]. The scatterplot matrix with prediction ellipses has proven to be a fast graphic analysis and a preliminary stage for a detailed statistical evaluation in our study.

Multivariate analyses were also used to examine the independent predictors for other clinical endpoints. Significant positive correlations between ROM and years in service, more frequent subjective complaints of the upper extremity with increasing age, higher VAS under strain with a higher BMI, and higher grip strength with the presence of a secondary occupation and/or physically demanding hobbies are particularly noteworthy. These findings are relevant for future investigations, since the relationships of independent variables to each other may disturb the identification of risk factors for WMSDs, acting as confounders. These relationships should therefore be considered when analyzing any WMSD-related outcomes.

From a statistical point of view, the study has provided good statistical pre-conditions with regard to the proportion of WMSDs in our collective of $56 \%$. As this proportion was close to the perfect balance between WMSD-positive and WMSD-negative cases, the power for group comparison was near to maximum for the given sample size. The very low p-values for effects in the analysis of grip strength (see Table 5) probably resulted from the increase in power due to the higher number of individual measurements $(2 \times 3$ measurements per subject, i.e. total $n=420$ in 70 subjects). However, the variance analysis employed in regression models with other simply measured values, lacks such amount of information regarding the variance of the measurements.

We intentionally tried to avoid the term "significant" in regard to this analysis, in order not to refer our reported p-values to the conventional $5 \%$ significance level which may be prone to misinterpretation $[54,69]$. Using the concept of hypothesis testing in the scientifically accurate way, setting a significance level would require a multiplicity correction for a number of pre-defined tests. Such explicit correction would, on the other hand, take away our flexibility to follow effects and relations in our data, which contains a complex network of endpoints and their predictors. For this reason, this secondary analysis was clearly explorative. This means that the p-values shown in the tables are not referred to any significance level. They rather provide continuous information of how effects are related or ranked according to their strengths. In this respect, Table 3 in the results section has to be considered as identification of two potential candidates for appropriate surrogate measures for WMSD prevalence. However, real evidence for the adequacy of any of these candidates for WMSD detection in clinical use has to be generated by a dedicated study.

The DASH score was considered to be the surrogate endpoint of choice for our primary analysis of WMSD prevalence among medical device manufacturing employees. The current analyses confirm that DASH still has to remain as the closer choice, when assessing the WMSD status of a population.

Regarding the limitations of our study, it should be noted that cross-sectional studies always represent a snapshot and no statement can be made about the duration of an existing WMSD. In particular, it is not possible to clearly distinguish between chronic, recurrent, or acute diseases. As in other studies, we also focused on a manageable number of potential risk factors, since an increasing number of predictors increases the probability of false positive effects, especially in smaller samples. This makes it difficult to assess these effects as a whole. Due to the single investigator approach, there is a risk of systematic error for over-sensitive detection of WMSDs, which is indicated by the higher number of diagnoses compared to symptoms in our study. On the other hand, the examination was performed by the same hand surgeon, which may have led to the diagnosis at an earlier stage than in a clinical setting. However, reducing the systematic error by a multiple investigator approach would have brought an inter-observer error into play, arising as a result of different teaching backgrounds and subjective assessments. Repeating the physical examination tests by having two investigators examine the same person was not an option for the authors A major reason for this is that most test results depend on the announcement of symptoms (pain, numbness, etc.) and subjects learn during follow-up examinations, which limits the objectivity of such study designs [53]. Even though the cross-sectional design of our study does not permit causal inference, the observed relations provide valuable evidence for further research and policy making. For further limitations with regard to the three occupational activities, we would like to refer to our previous publication [17]. 


\section{Conclusions}

Methods used to collect, process and interpret data on WMSDs are extremely heterogeneous, so the comparability between studies is poor. This study evaluated survey methods and assessment tools for the detection of upper extremity WMSDs and its associations in a healthy working population.

While the most frequently used questionnaires focus on subjective complaints that do not seem to be related to WMSDs, the DASH questionnaire could prove to be an efficient screening method. However, the gold standard for the detection of WMSDs, but also for the derivation of prophylactic, therapeutic and rehabilitative measures, is still the standardised physical examination based on a predetermined set of diagnostic criteria.

Our analysis has not identified any risk factors for WMSDs in the study data. Possibly, the effects of investigated risks were too small to be detected by our relatively small study sample.

In order to make epidemiological research on upper extremity WMSDs more comparable, a uniform study design in regard to endpoint selection is recommended. We hope that the methodological results of our work will help other researchers to obtain more efficient and consistent tools for the research on upper extremity WMSDs.

\section{Abbreviations}

2PD

Two-point discrimination

BMI

Body mass index

CTD

Cumulative trauma disorders

DASH

Disabilities of the Arm, Shoulder and Hand

E/F

Extension / flexion

ICF

International Classification of Functioning, Disability and Health

NMQ

Nordic Musculoskeletal Questionnaire

oOs

Occupational overuse syndromes

PPB Test

Purdue Pegboard Test

ROM

Range of motion

RSI

Repetitive strain injuries

S/P

Supination / pronation

$\mathrm{U} / \mathrm{R}$

Ulnar / radial abduction

VAS

Visual Analogue Scale

WMSD 


\section{Declarations}

\section{Ethics approval and consent to participate}

This study was performed in accordance with the Declaration of Helsinki and was approved by the Ethics Committee of the State Medical Council of Baden-Wuerttemberg, Jahnstrasse 40, 70597 Stuttgart, Germany on January 24th, 2017 (project number F-2017-005). All participants in this study provided personal and written consent.

\section{Consent for publication}

Not applicable.

\section{Availability of data and materials}

All templates for questionnaires and survey forms are available upon request from the corresponding author. The blinded data sets are under lock and key at the Aesculap AG Health Centre and are not available.

\section{Competing interests}

VB is an employee of the Department of Medical Scientific Affairs at Aesculap AG, Tuttlingen,

\section{Funding}

Part of this work was supported by Aesculap AG, Tuttlingen/Germany. The funder did not have a role in designing, conducting, analyzing, interpreting or reporting the results of the research.

\section{Authors' contributions}

The following authors have made substantial contributions to the conception: OL, JM and TL; design of the work: TL and JM; statistics: OL and VB, acquisition and analysis: TL and OL; interpretation of data: OL, JM and VB, drafting and revision of the work: OL and VB. All the authors read and approved the final manuscript.

\section{Acknowledgements}

The authors thank all participants in this study and the staff involved in the implementation of the study, in particular Dr. rer. nat. Petra Baumann, MBA, Aesculap AG; Mrs. Stefanie Latuske, Mrs. Mevlüde Orhan and Mrs. Ulrike Hofer, Health Center, Aesculap AG.

\section{References}

1. Bernard BP. Musculoskeletal disorders and workplace factors: a critical review of epidemiologic evidence for work-related musculoskeletal disorders of the neck, upper extremity and low back. US Department of Health and Human Services. NIOSH publication 1997;97-141.

2. Baldwin ML, Butler RJ. Upper extremity disorders in the workplace: costs and outcomes beyond the first return to work. J Occup Rehabil. 2006;16:303-23. 
3. Buckle PW, Devereux JJ. The nature of work-related neck and upper limb musculoskeletal disorders. Appl Ergon. 2002;33:207-17.

4. Palmer K, Coggon D, Cooper C, Doherty M. Work related upper limb disorders: getting down to specifics. Ann Rheum Dis. 1998;57:445-6.

5. Snashall D. ABC of work-related disorders- hazards of work. BMJ 1996;313:161.

6. Melhorn JM. Cumulative trauma disorders and repetitive strain injuries. Clin Orthop Relat Res. 1998;351:107-126.

7. Backhans M, Stjernschantz Forsberg J, Lager A, editors. Folkhälsorapport 2015 [Public health report 2015]. Stockholm: Center of Epidemiology and Community Medicine: Stockholm County Council; 2015.

8. Kumar VK, Kumar SP, Baliga MR. Prevalence of work-related musculoskeletal complaints among dentists in India: A national cross-sectional survey. Indian J Dent Res. 2013;24:428-38.

9. Van Eerd D, Beaton D, Cole D, Lucas J, Hogg-Johnson S, Bombardier C. Classification systems for upper-limb musculoskeletal disorders in workers: a review of the literature. J Clin Epidemiol. 2003;56:925-36.

10. Da Costa JT, Baptista JS, Vaz M. Incidence and prevalence of upper-limb work related musculoskeletal disorders: A systematic review. 2015;51:635-44.

11. Hoy DG, Smith E, Cross M, Sanchez-Riera L, Buchbinder R, Blyth FM. The global burden of musculoskeletal conditions for 2010: an overview of methods. Annals of the rheumatic diseases. 2014;73:982-989.

12. Jensen LK, Hansen HB. Working environment. Many work impediments among personnel in operating room. Sygeplejersken. 1986;86:4-10.

13. Mather L, Ropponen A, Mittendorfer-Rutz E, Narusyte Health, work and demographic factors associated with a lower risk of work disability and unemployment in employees with lower back, neck and shoulder pain. BMC Musculoskelet Disord. 2019;20:622.

14. Warren N, Dillon C, Morse T, Hall C, Warren A. Biomechanical, psychosocial, and organizational risk factors for WRMSD: population-based estimates from the Connecticut upper-extremity surveillance project (CUSP). J Occup Health Psychol.2000;5:164-81.

15. Gerr F, Fethke NB, Merlino L, Anton D, Rosecrance J, Jones MP. A prospective study of musculoskeletal outcomes among manufacturing workers: Effects of physical risk factors. Hum Factors. 2014,56,112-130.

16. Burdorf A, Sorock G. Positive and negative evidence for risk factors of work-related back disorders. Scand J Work Environ Health 1997;23:243-256.

17. Lotter O, Lieb T, Breul V, Molsner J. Is Repetitive Workload a Risk Factor for Upper Extremity Musculoskeletal Disorders in Surgical Device Mechanics? A Cross-Sectional Analysis. Int J Environ Res Public Health. 2020; doi:10.3390/ijerph17041383.

18. Germann G, Harth A, Wind G, Demir E. Standardisation and validation of the German version 2.0 of the Disability of Arm, Shoulder, Hand (DASH) questionnaire. Unfallchirurg 2003;106:13-9.

19. Hudak PL, Amadio PC, Bombardier C. Development of an upper extremity outcome measure: The DASH (disabilities of the arm, shoulder and hand). Am J Ind Med. 1996;29:602-8.

20. Ryf C. Weymann, A. The neutral zero method - A principle of measuring joint function. Injury 1995;26:1-11.

21. Sherman EMS. The Purdue Pegboard Test. In: Strauss E, Sherman EMS, Spreen O, editors. A Compendium of Neuropsychological Tests: Administration, Norms, and Commentary. 3rd ed. Oxford: Oxford University Press; 2006. p. 1068-79.

22. Golden CJ, Espe-Pfeifer P, Wachsler-Felder J. Neuropsychological Interpretations of Objective Psychological Tests. 1 st ed. Heidelberg: Springer; 2006.

23. Waris P, Kuorinka I, Kurppa K, Luopajärvi T, Virolainen M, Pesonen K, Nummi J, Kukkonen R. Epidemiologic screening of occupational neck and upper limb disorders. Methods and criteria. Scand J Work Environ Health. 1979;5:25-38.

24. Finkelstein, H. Stenosing tendovaginitis at the radial styloid process. J. Bone Joint Surg. 1930;12:509-40.

Page $14 / 18$ 
25. Sheon RE, Moskowitz RW, Goldberg VM. Soft Tissue Rheumatic Pain: Recognition, Management, Prevention. 2nd ed. Philadelphia: Lea \& Febiger; 1988.

26. Phalen GS. The carpal tunnel syndrome: Seventeen years' experience in diagnosis and treatment of 654 hands. J. Bone Joint Surg. 1966;48:211-228.

27. Leclerc A, Landre M, Chastang J, Niedhammer I, Roquelaure Y. Study Group on Repetitive Work. Upper-limb disorders in repetitive work. Scand J Work Environ Health. 2001;27:268-78.

28. Lang TA, Secic M. How to report statistics in medicine. 2nd ed. Philadelphia: American College of Physicians; 2006.

29. Hunsaker FG, Cioffi DA, Amadio PC, Wright JG, Caughlin B. The American academy of orthopaedic surgeons outcomes instruments: Normative values from the general population. J Bone Joint Surg Am. 2002;84:208-215.

30. Guenther CM, Buerger A, Rickert M, Crispin A, Schulz CH. Grip strength in healthy caucasian adults: Reference values. J Hand Surg Am. 2008;33:558-65.

31. Agnew J, Bolla-Wilson K, Kawas CH, Bleeker ML. Purdue Pegboard age and sex norms for people 40 years old and older. Develop Neuropsych. 1988;4:29-35.

32. Confidence and Prediction Ellipses.

https://support.sas.com/documentation/cdl/en/procstat/66703/HTML/default/viewer.htm\#procstat_corr_details19.htm. Accessed 21 July 2013.

33. Jester A, Harth A, Rauch J, Germann G. DASH Data of Non-Clinical versus Clinical Groups of Persons - A Comparative Study of T-norms for Clinical Use. Handchir Mikrochir Plast Chir. 2010;42: 55-64.

34. Van Eerd D, Beaton D, Cole D, Lucas J, Hogg-Johnson S, Bombardier C. Classification systems for upper-limb musculoskeletal disorders in workers: A review of the literature. J Clin Epidemiol. 2003;56:925-36.

35. Lipscomb HJ, Epling CA, Pompeii LA, Dement JM. Musculoskeletal symptoms among poultry processing workers and a community comparison group: Black women in low-wage jobs in the rural South Am J Ind Med. 2007;50:327-38.

36. Macdonald F, Waclawski E. Upper limb disorders among coopers in the Scotch whisky industry. Occup Med. 2006;56:23236.

37. Ohlsson K, Attewell RG, Pålsson B, Karlsson B, Balogh I, Johnsson B, Kilbom A, Kuorinka IA, Silverstein BA, Sjogaard G, Viikari-Juntura ER. Repetitive industrial work and neck and upper limb disorders in females. Am J Ind Med. 1995;27:73147.

38. Ohlsson K, Hansson GA, Balogh I, Stroemberg U, Pålsson B, Nordander C, Rylander L, Skerfving S. Disorders of the neck and upper limbs in women in the fish processing industry. Occup Environ Med. 1994;51:826-32.

39. Bovenzi M, Zadini A, Franzinelli A, Borgogni F. Occupational musculoskeletal disorders in the neck and upper limbs of forestry workers exposed to hand-arm vibration. Ergonomics 1991;34:547-62.

40. Punnett L, Robins JM, Wegman DH, Keyserling WM. Soft tissue disorders in the upper limbs of female garment workers. Scand. J Work Environ Health. 1985;11:417-25.

41. Luopajärvi T, Kuorinka I, Virolainen M, Holmberg M. Prevalence of tenosynovitis and other injuries of the upper extremities in repetitive work. Scand J Work Environ Health 1979;5:48-55.

42. Ohlander J, Keskin MC, Weiler S, Stork J, Radon K. Snap-fits and upper limb functional limitations in German automotive workers. Occup Med. 2016;66:471-77.

43. Taspinar O, Kepekci M, Ozaras N, Aydin T, Guler M. Upper extremity problems in doner kebab masters. J Phys Ther Sci. 2014;26:1433-6.

44. Ghosh T, Das B, Gangopadhyay S. A comparative ergonomic study of work-related upper extremity musculoskeletal disorder among the unskilled and skilled surgical blacksmiths in West Bengal, India. Indian J Occup Environ Med. 2011;15:127-32.

45. Hembecker PK, Reis DC, Konrath AC, Gontijo L, Merino EA. Investigation of musculoskeletal symptoms in a manufacturing company in Brazil: A cross-sectional study. Braz J Phys Ther. 2017;21:175-83.

Page $15 / 18$ 
46. Gold JE, d'Errico A, Katz JN, Gore R, Punnett L. Specific and non-specific upper extremity musculoskeletal disorder syndromes in automobile manufacturing workers. Am J Ind Med 2009;52:124-32.

47. Viikari-Juntura E. Neck and upper limb disorders among slaughterhouse workers. An epidemiologic and clinical study. Scand J Work Environ Health. 1983;8:283-90.

48. Shiri R, Viikari-Juntura E, Varonen H, Heliövaara M. Prevalence and determinants of lateral and medial epicondylitis: A population study. Am J Epidemiol. 2006;164:1065-74.

49. Wolf JM, Mountcastle S, Burks R, Sturdivant RX, Owens BD. Epidemiology of lateral and medial epicondylitis in a military population. Mil Med 2010;175:336-39.

50. Crouch T. Obtaining an Accurate Diagnosis. In: Crouch T, editor. Carpal Tunnel Syndrome and Repetitive Stress Injuries: The Comprehensive Guide to Prevention, Treatment, and Recovery. 2nd ed. Berkeley: North Atlantic Books; 1992. p. $23-7$.

51. Kilbom S, Armstrong T, Buckle P, Fine L, Hagberg M, Haring-Sweeney M, Martin B, Punnett L, Silverstein B, Sjøgaard G, Theorell T, Viikari-Juntura E. Musculoskeletal Disorders: Work-related Risk Factors and Prevention. Int J Occup Environ Health. 1996;2:239-246.

52. Burdorf A, van der Beek AJ. In musculoskeletal epidemiology are we asking the unanswerable in questionnaires on physical load? Scand J Work Environ Health. 1999;25: 81-3.

53. Hansson G-Å, Balogh I, Unge Byström J, Ohlsson K, Nordander C, Asterland P, Sjölander S, Rylander L, Winkel J, Skerfving S. Questionnaire versus direct technical measurements in assessing postures and movements of the head, upper back, arms and hands. Scand J Work Environ Health. 2001;27:30-40.

54. Altman DG, Bland JM. Absence of evidence is not evidence of absence. BMJ. 1995;311:485.

55. Ostlie K, Franklin RJ, Skjeldal OH, Skrondal A, Magnus Assessing Physical Function in Adult Acquired Major Upper-Limb Amputees by Combining the Disabilities of the Arm, Shoulder and Hand (DASH) Outcome Questionnaire and Clinical Examination. Arch Phys Med Rehabil. 2011;92:1636-45.

56. Dickinson CE, Campion K, Foster AF, Newman SJ, Orourke AMT, Thomas PG. Questionnaire development - An examination of the Nordic musculoskeletal questionnaire. Appl Ergon. 1992;23:197-201.

57. Occupational Safety and Health Administration, Washington. 2016. https://www.osha.gov/SLTC/etools/hospital/sonography/sonography.html

58. Punnett L, Wegman DH. Work-related musculoskeletal disorders: The epidemiological evidence and the debate. J Electromyograph Kinesiol. 2004;14:13-23.

59. Lipscomb HJ, Epling CA, Pompeii LA, Dement JM. Musculoskeletal symptoms among poultry processing workers and a community comparison group: Black women in low-wage jobs in the rural South. Am J Ind Med. 2007;50:327-38.

60. Davis TR. Do repetitive tasks give rise to musculoskeletal disorders? Occup Med. 1999;49:257-8.

61. Berberoglu U., Tokuc B. Work-related musculoskeletal disorders at two textile factories in Edirne, Turkey. Balkan Medical Journal. 2013;30:23-7.

62. Wang PC, Rempel D, Harrison R, Chan J, Ritz B. Work-organizational and personal factors associated with upper body musculoskeletal disorders among sewing machine operators. Occupational and Environmental Medicine. 2007;10:1-8.

63. Pelissier C, Fontana L, Fort E. Occupational risk factors for upper-limb and neck musculoskeletal disorder among healthcare staff in nursing homes for the elderly in France. Industrial Health. 2014;52:334-46.

64. Moodley R, Naidoo S. The prevalence of musculoskeletal disorders among dentists in KwaZulu-Natal. South African Dental Journal. 2015;70:98-103.

65. Alshagga MA, Nimer AR, Yan LP, Ibrahim IAA, Al-Ghamdi SS, Al-Dubai SAR. Prevalence and factors associated with neck, shoulder and low back pains among medical students in a Malaysian Medical College. BMC Research Notes. 2013;6:1-7.

66. Rodrigues EV, Gomes ARS, Tanhoffer AIP, Leite N. Effects of exercise on pain of musculoskeletal disorders: a systematic review. Acta Ortopédica Brasileira. 2014;22:334-8. 
67. Mekonnen TH, Abere G, Olkeba SW. Risk Factors Associated with Upper Extremity Musculoskeletal Disorders among Barbers in Gondar Town, Northwest Ethiopia, 2018: A Cross-Sectional Study. Pain Res Manag.2019; doi:

$10.1155 / 2019 / 6984719$.

68. Sternberg A, Bethge M. Measuring work functioning in individuals with musculoskeletal disorders with reference to the International Classification of Functioning, Disability, and Health: a systematic literature review. Int J Rehabil Res. 2018;41:97-109.

69. Amrhein V, Greenland S, McShane B. Scientists rise up against statistical significance. Nature. 2019;567:305-7.

\section{Figures}

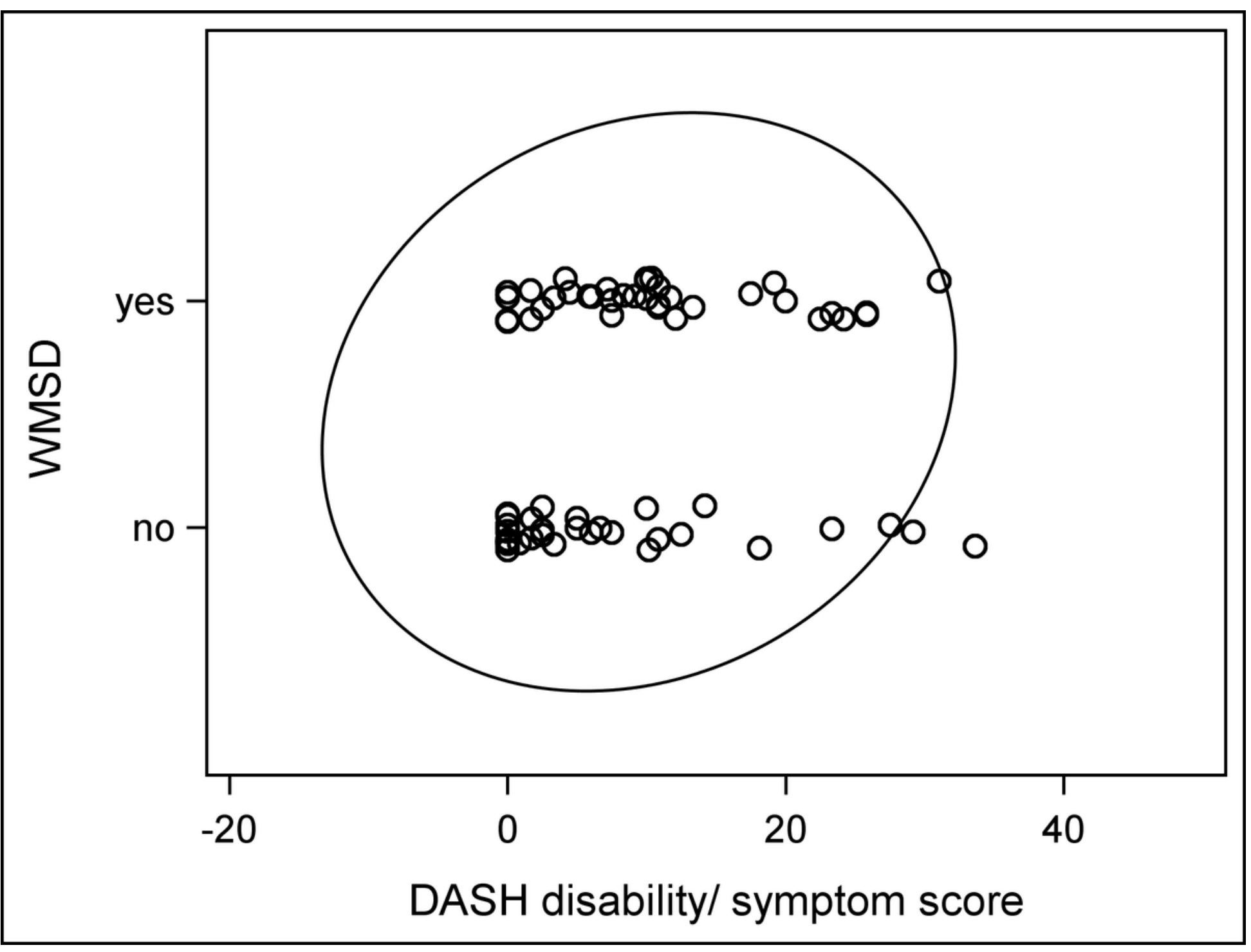

Figure 1

Prediction ellipse for graphical representation of the correlation between WMSDs and the DASH score Legend for Figure 1: $\mathrm{N}=$ 67 , Pearson $r=0.16, p=0.182$, Spearman $r=0.23 p=0.056$

\section{Supplementary Files}

This is a list of supplementary files associated with this preprint. Click to download. 
- DASHquestionnaireGermanoriginal.pdf

- DASHquestionnaireEnglishversion.pdf

- BasicdataquestionnaireGermanoriginal.docx

- BasicdataquestionnaireEnglishtranslation.docx 